\title{
Applications of digital technology for the morphological study of C3-C7 vertebral arch pedicle in children
}

\author{
S. Zhang 1, 2, X. Wang 1, 2, X. Ren ${ }^{3}$, Z. Li ${ }^{1}$, Y. Zhang ${ }^{2}$ \\ ${ }^{1}$ Teaching and Researching Department of Human Anatomy, Basic Medical College of Inner Mongolia Medical University, \\ Inner Mongolia, China \\ 2Digital Medical Centre of Inner Mongolia Medical University, Inner Mongolia, China \\ ${ }^{3}$ Department of Endocrinology, Affiliated Hospital of Inner Mongolia Medical University, Inner Mongolia, China
}

[Received: 8 November 2016; Accepted: 7 December 2016]

Background: This study aims to investigate the morphological characteristics and developmental patterns of the vertebral arch pedicle (VAP) in the lower cervical vertebrae of children, and to subsequently help guide the implantation of the pedicle screw.

Materials and methods: The cervical vertebral multi-slice computed tomography (MSCT) data of 60 paediatric patients aged 4-12 years were collected and grouped. A reconstructed 3-dimensional model measured the following: pedicle width $(P W)$, pedicle height ( $P H)$, pedicle osseous channel (POCL), pedicle transverse angle (PTA, namely the $E$ angle), and pedicle sagittal angle (PTA, namely the F angle). Results: We calculated the ratio of PWIPH (I value) to assess the statistical difference between age groups and segments. The PW, PH, POCL, and E angles exhibited an overall increasing trend with increasing age. The $P W, P H$, and POCL of each vertebra in group $C$ showed statically significant differences compared to groups $A$ and $B(p<0.05)$. The PW of different vertebral sequences in each group showed a gradually increasing trend. The intervertebral $F$ angle among different groups showed the biggest difference, a change from positive values to negative values gradually, among which the negative value of C7's F angle was the largest. The I value exhibited an overall decreasing trend with increasing age. Conclusions: The morphological characteristics and development of the different pedicle segments exhibited obvious patterns. In the lower cervical vertebrae of children over 7 years of age, the vertebrae had the feasibility for the implantation of pedicle screws. (Folia Morphol 2017; 76, 3: 426-432)

Key words: lower cervical vertebrae, vertebral arch pedicle, digital measurement, children

\section{INTRODUCTION}

In recent years, the pedicle screw internal fixation technology, characterised by its stable and firm biomechanical features, has been used in the treat- ment of vertebral injuries and diseases in paediatric patients. Paediatric vertebral injuries occur mostly in the cervical vertebrae [12] as the vertebral arch pedicle (VAP) of paediatric cervical vertebrae is more fine and

Address for correspondence: Dr. Z. Li, Teaching and Researching Department of Human Anatomy, Basic Medical College of Inner Mongolia Medical University, Hohhot 010110, Inner Mongolia, China, tel: +86 471 6657562, fax: +86 471 6657548, e-mail: zhijunlidc@126.com 
fragile. In addition to this, the vertebrae of paediatric patients are in the growth and development stage, making the applications of this technology on paediatric cervical vertebrae limited. Currently, researchers hypothesized that the C2 VAP in children under 10 years of age has the morphological feasibility of implanting 3.5-mm pedicle screws [7]. Clinical reports provide more details about the internal pedicle screw fixation in the upper cervical vertebrae of children $[2,4]$. Treatments in lower cervical vertebral injury and cancer reconstruction in children mainly use such conventional methods as trans-lateral mass screw fixation or translaminar screw fixation $[3,5]$. However, these traditional methods show no obvious biomechanical advantages. Previous studies examined whether the morphologies of the lower cervical vertebrae in children are suitable for screw implantation [7, 18]. However, these studies normally used cadavers or the methods for 2-dimensional (2D) image acquisition. The applications of 3-dimensional (3D)-reconstructed digital technology for VAP anatomic morphology, development pattern, and screw implantation feasibility in the lower cervical vertebrae of different-age children are rarely used. Based on the computed tomography (CT) thin-layer imaging data in the lower cervical vertebrae of children 4-12 years of age, the purpose of our study was to use the MIMICS (Materialise's Interactive Medical Image Control System) software to reconstruct a 3D cervical vertebral model, measure the pedicle screw implantation-related parameters, and summarise its morphological features and development patterns. The results of our study can provide the morphological basis for the pedicle screw implantation in the lower cervical vertebrae of children and assess the feasibility of the $3.5-\mathrm{mm}$ screws.

\section{MATERIALS AND METHODS}

\section{General information}

Between September 2008 and June 2013, we collected MSCT data from paediatric patients from the Department of Radiology in the First and Second Affiliated Hospitals of Inner Mongolia Medical University. We included patients who met the following inclusion criteria: (1) the scan used the GE Light QX/I 64-slice spiral CT; (2) the scanning range included the area encompassing the paropia to the superior aperture of the thorax (including the skull base to T1); (3) the scanning lines were vertical to the body's central axis; and (4) the scanning parameters included the following: layer thickness: $1.25 \mathrm{~mm}$; pitch: $1.25 \mathrm{~mm}$; reconstruction slice thickness and spacing: $0.625 \mathrm{~mm}$; FOV: $30 \times 30 \mathrm{~cm}$; and matrix:
$512 \times 512$ dpi. We excluded patients with cervical vertebral trauma, deformity, and significant neurological signs and symptoms. Using the eligibility criteria, the authors identified a total of 60 paediatric patients, between 4 to 12 years of age. We divided the patients into three groups with an age interval of 3 years: 4-year-old group (group A), 7-year-old group (group B), and 10-12-yearold group (group C), with 20 patients in each group. The authors measured both pedicle sides of each patient, for a total of 40 sets of data. The mean value of multiple measurements was used to avoid artificial errors. This study was conducted in accordance with the declaration of Helsinki. This study was conducted with approval from the Ethics Committee of Inner Mongolia Medical University. Written informed consent was obtained from all participants' guardians.

\section{Measurement indexes}

We defined pedicle width (PW) as the shortest distance between the outer edges of the lateral and interior cortex at the VAP gorge. Pedicle height (PH) is the shortest distance between the outer edges of the superior and inferior cortex at the pedicle gorge. The pedicle osseous channel (POCL) is the shortest distance between the vertebral posterior cortex and the vertebral anterior cortex along the pedicle axis. The $\mathrm{E}$ angle is the angle between the pedicle axis and the corresponding vertebral sagittal plane. The $F$ angle is the angle between the pedicle axis and the corresponding vertebral plane at the caudal. When the pedicle axis pointed to the superior endplate, the $F$ angle was positive; otherwise, it was negative.

\section{Measurement methods}

Data were imported into MIMICS (Materialise Co., Belgium) with the DICOM format for 3D reconstruction with the appropriate Hounsfield unit (HU) window. The corresponding pedicle axis was then selected and adjusted in the reconstruction model. From the three axial views the intersections with the vertebral anterior cortex and the vertebral posterior cortex were calibrated (namely point $\mathrm{A}$ and $\mathrm{B}$, respectively). Using the 3D measurement tools, $\mathrm{PW}, \mathrm{PH}$, and $\mathrm{POCL}$ were measured on the reconstructed $3 \mathrm{D}$ model. The cross and sagittal VAP planes were then selected to measure the $E$ and $F$ angles, respectively. If these angles could not be measured, the model was imported into the 3-Matic software (Materialise Co., Belgium) and projected onto the cross and sagittal pedicle planes for measurement (Fig. 1). 


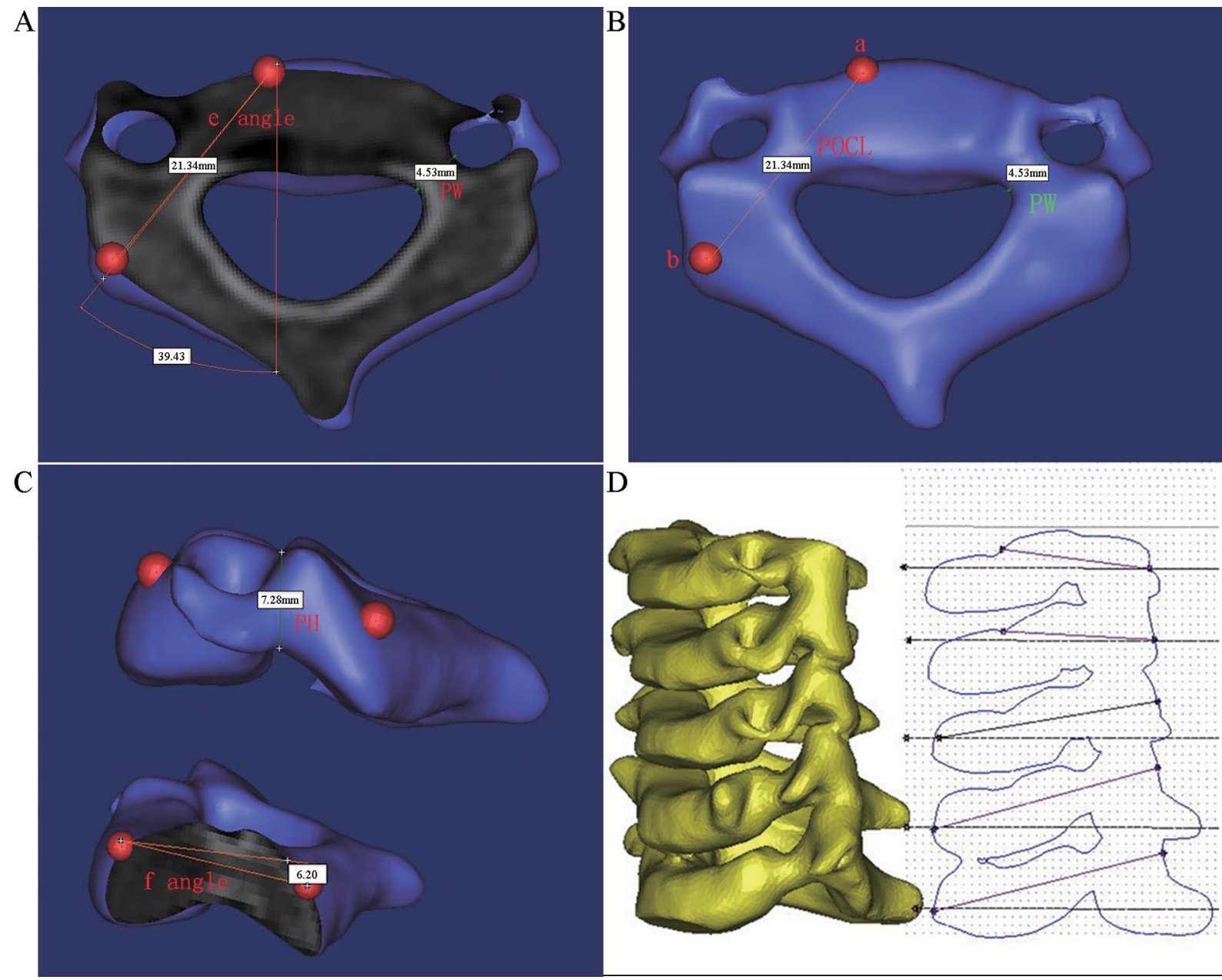

Figure 1. A. Pedicle osseous channel (POCL) and pedicle width (PW) measurement using three-dimensional measurement tools; $B$. E angle measurement on the cross pedicle plane; $\mathbf{C}$. Pedicle height $(\mathrm{PH})$ and $\mathrm{F}$ angle measurement; $\mathbf{D}$. Changes of $\mathrm{F}$ angle showed by the projection of $\mathrm{C} 3 \sim \mathrm{C} 7$ on the pedicle axial plane (C3 and $\mathrm{C} 4$ were positive, $\mathrm{C} 5 \sim \mathrm{C} 7$ were negative).

\section{Statistical methods}

All data were imported into Microsoft Excel (Microsoft, Redmond, WA) and SPSS 16.0 (IBM Inc., New York, NY) for sorting and statistical analysis. Data are expressed as the mean \pm standard deviation. A one-way ANOVA was used to identify any differences between the groups. The Student-NewmanKeuls (SNK) method was used to identify significantly different sample means. A p-value $<0.05$ was considered statistically significant.

\section{RESULTS}

\section{Pedicle width}

The overall PW exhibited an increasing trend with increasing age. Patients in group $C$ showed statistically significant differences compared to groups $A$ and $B(p<0.05)$. There was no difference between groups $A$ and $B(p>0.05)$. PW in each group exhibited a gradually increasing trend with the vertebral sequence. There was a significant difference between $C 4$ and $C 7$ in group $A(p<0.05)$; in group $B$, there were significant differences between $C 3$ and $C 5 / 6 / 7$ and between $C 4$ and $C 6 / 7$ $(p<0.05)$; in group $C$ there was a significant difference between $C 4$ and $C 7(p<0.05)$. The values of C3 and C4 in each group were relatively smaller (Table 1).

\section{Pedicle height}

The overall PH exhibited an increasing trend with increasing age. There were significant differences between $C 3, C 5$, and $C 6$ in groups $A$ and $B(p<0.05)$. While there was a difference in each vertebra between groups $C$ and $A / B$, this difference was not significant $(p>0.05)$. There were no differences between the vertebrae in the same group $(p>0.05)$ (Table 1$)$. 


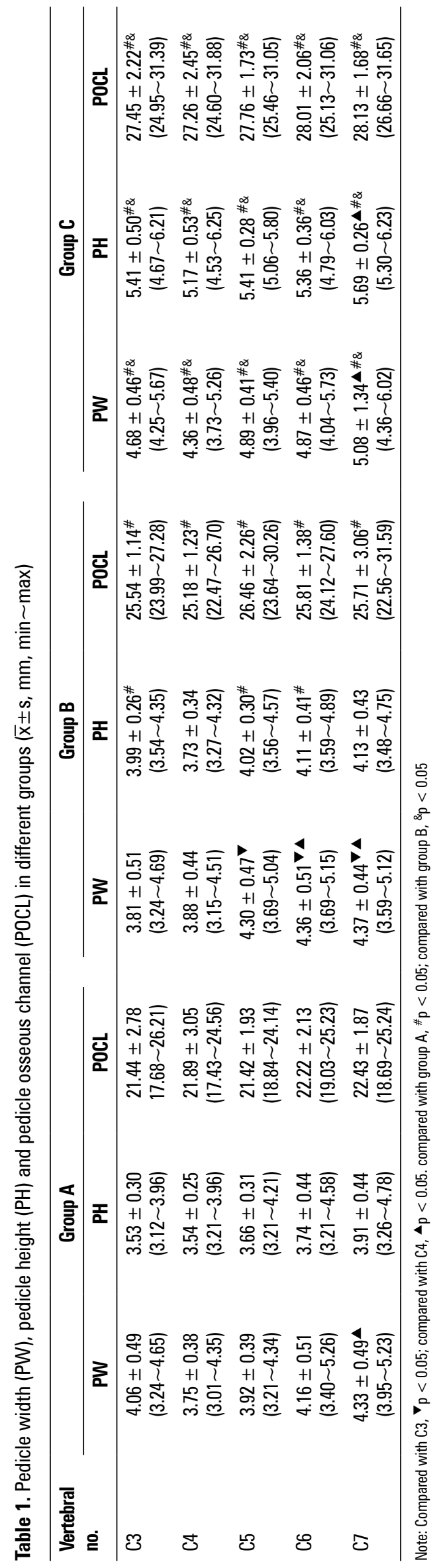

\section{Pedicle osseous channel}

The overall POCL exhibited an increasing trend with increasing age. There were significant differences among the three groups $(p<0.05)$; however, there were no significant differences between the vertebrae in the same group (Table 1).

\section{E angle}

The overall $\mathrm{E}$ angle exhibited an increasing trend with increasing age; however, the differences among the three groups were not statistically significant. There were significant differences in $\mathrm{C} 3$ and $\mathrm{C} 4$ between groups $A$ and $C / B(p<0.05)$, and in $C 5$ between groups $C$ and $A / B$; however, there were no differences between the vertebrae in the same group (Table 2).

\section{F angle}

The overall $F$ angle did not change much with increasing age. There were significant differences in C3 between groups B and A/C $(p<0.05)$ and there were differences in $\mathrm{C} 5$ between groups $A$ and $C$. The biggest differences occurred between the vertebrae in the same group. The vertebrae gradually decreased from $\mathrm{C} 5$ to $\mathrm{C7}$; changing from positive to negative. The biggest negative value occurred at the $\mathrm{F}$ angle at C7 (Table 2, Fig. 1).

\section{I value}

The overall I value exhibited a decreasing trend with increasing age. There were significant differences in $C 3$ between groups $A$ and $B / C(p<0.05)$ and there were differences in $\mathrm{C} 4$ to $\mathrm{C} 7$ between groups $\mathrm{C}$ and $A / B$. The I values in $C 3$ to $C 7$ in each group changed from bigger than 1.0 to smaller than 1.0 gradually; however, there were no significant differences between the vertebrae in the same group (Table 3).

\section{DISCUSSION}

The pedicle screw internal fixation technology, characterised by its stable and firm biomechanical features, is widely used in treating cervical vertebral injuries in adults [9, 10, 20]. Ruf and Harms [15] performed the hemivertebral resection plus pedicle screw internal fixation in 28 paediatric patients with congenital scoliosis (1-6 years old) and achieved satisfactory results. In another study, 19 paediatric patients (1-2 years old) underwent pedicle screw internal fixation for their thoracic and lumbar injuries [16]. Thus, this technology is used in paediatric 


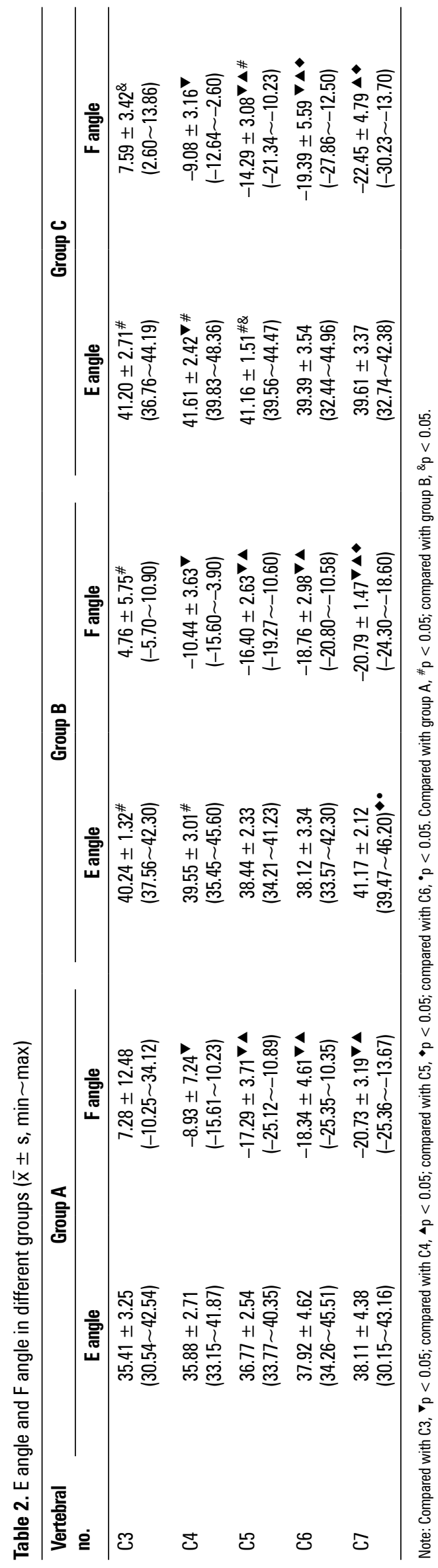

Table 3. I values in different groups $(\bar{x} \pm s, \min \sim \max )$

\begin{tabular}{lccc}
\hline $\begin{array}{l}\text { Vertebral } \\
\text { no. }\end{array}$ & Group A & Group B & Group C \\
\hline C3 & $1.15 \pm 0.11$ & $0.95 \pm 0.11$ & $0.87 \pm 0.09$ \\
& $(1.03 \sim 1.31)$ & $(0.81 \sim 1.14)^{\#}$ & $(0.68 \sim 0.97)^{\#}$ \\
C4 & $1.09 \pm 0.07$ & $1.04 \pm 0.12$ & $0.85 \pm 0.08$ \\
& $(1.01 \sim 1.22)$ & $(0.87 \sim 1.27)$ & $(0.70 \sim 0.99)^{\# \&}$ \\
C5 & $1.13 \pm 0.10$ & $1.07 \pm 0.65$ & $0.91 \pm 0.09$ \\
& $(1.01 \sim 1.30)$ & $(0.94 \sim 1.19)$ & $(0.68 \sim 0.99)^{\# \&}$ \\
C6 & $1.12 \pm 0.10$ & $1.06 \pm 0.07$ & $0.91 \pm 0.10$ \\
& $(1.03 \sim 1.28)$ & $(0.94 \sim 1.16)$ & $(0.78 \sim 1.10)^{\# \&}$ \\
C7 & $1.11 \pm 0.09$ & $1.06 \pm 0.04$ & $0.89 \pm 0.10$ \\
& $(1.01 \sim 1.30)$ & $(1.01 \sim 1.13)$ & $(0.70 \sim 1.06)^{\# \&}$ \\
\hline
\end{tabular}

Note: Compared with group $A$, ${ }^{\#} p<0.05$; compared with group $B$, \& $<0.05$

patients. In recent years, this technology has been widely used in the vertebral injuries of paediatric patients and has proven to be feasible [11]. However, reported studies discuss the thoracolumbar internal fixation of paediatric patients $[17,19]$ and rarely address the cervical vertebral injury. When treating cervical vertebral diseases, the pedicle screws internal fixation might exhibit better biomechanical stability than traditional methods. However, there are more difficulties when pedicle screws are implanted on the cervical vertebrae of paediatric patients $[6,8]$. Furthermore, because children are in the growth and development stage, their pedicles are fine and fragile. Thus, to identify development patterns, 3D morphological studies should be performed to assess the features of pedicles at different ages. Once identified, we can investigate the feasibility of $3.5-\mathrm{mm}$ pedicle screw implantation.

In this study, the overall values of $\mathrm{PW}, \mathrm{PH}$, and POCL exhibited an increasing trend with the vertebral sequence increasing; however, the intervertebral differences were not significant. The overall conditions showed an increasing trend with increasing age. PW, $\mathrm{PH}$, and POCL of each vertebra in group $\mathrm{C}$ were statistically bigger than those in groups $A$ and $B$. This may indicate that the cervical vertebrae in children grow faster between the ages of 10 to 12 years. PW and $\mathrm{PH}$ showed an increasing trend with increasing age, with $\mathrm{PH}$ exhibiting faster growth than PW. The I value gradually changed from $>1.0$ to $<1.0$; therefore, $\mathrm{PW}$ in group $\mathrm{A}$ was greater than $\mathrm{PH}$. The shape of the cross section was transverse oval or near round. $\mathrm{PW}$ in group $B$ and $C$ were smaller than $\mathrm{PH}$, thus the shape of the cross section was round or longitudinal oval. 
As age increased, the cross section shape appeared closer to a longitudinal oval shape. The overall POCL showed an increasing trend with increasing age. The differences in each cervical vertebra between the three groups were statistically significant, indicating that the overall growth of the vertebrae was obvious with increasing age. The overall $E$ angle increased as the vertebral sequence increased; however, the intervertebral differences were not significant. The changes in the F angle were large, which gradually changed from positive to negative. This increase may be due to the formation of cervical curvature and protrusion in this age segment.

Successful implantation of the pedicle screw refers to the correct position and orientation of the screw(s), the confirmed depth of the screw(s), and the selection of the pedicle screw(s) with the appropriate diameter. The ratio of the screw diameter to the pedicle diameter determines the stability and safety. The gripping force of the pedicle screw is positively proportional to the diameter and length of the pedicle screw implanted. In adults, it is believed that the ratio of $4 / 5$ could achieve more stable biomechanical effects of the internal fixation. Too thick of a screw might break through the pedicle and injure the surrounding tissues due to the fragility of adult bones. However, the cortical flexibility in children is strong. Polly et al. [13] reported that the pedicle elasticity of children is good, and therefore, the pedicle screw diameter could be $115 \%$ of the transverse diameter of the target pedicle. Rinella et al. [14] gradually increased the pedicle screw diameters in 9-year-old children by continuously implanting different types of screws. The pedicle screw diameter in paediatric patients was increased by $74 \%$ and $24 \%$, respectively, in the inner and outer transverse. Thus knowing the corresponding pedicle can help determine the ideal screw diameter. Meanwhile, the "slightly thicker" screw(s) can be used according to such characteristics of paediatric pedicles, bigger flexibility and expansion can increase the antiwithdrawal and anti-distortion force.

The PW in group B was larger than PH. Thus, the screw diameter depended primarily on its $\mathrm{PH}$; the mean $\mathrm{PH}$ values ranged from $3.53 \mathrm{~mm}$ to $3.91 \mathrm{~mm}$ from $\mathrm{C} 3$ to $\mathrm{C} 7$. Because of the ductility and elasticity of the paediatric pedicle, screw implantation is feasible. However, the risks are also high. In addition to this, individual differences are also significant. Therefore, preoperative carefully designed screw programmes and intraoperative technical skills are necessary. PW in group $\mathrm{C}$ was smaller than $\mathrm{PH}$. Hence, the screw diameter depended on PW; the mean PW values ranged from $3.81 \mathrm{~mm}$ to $5.08 \mathrm{~mm}$. Thus, implanting the $3.5-\mathrm{mm} \mathrm{screw}(\mathrm{s})$ in our population is feasible. However, additional studies are recommended to determine the generalisability of this study. Fujimori et al. [1] collected data on a large number of clinical surgeries and confirmed the feasibility of pedicle screw implantation in infants and children. However, the surgical complications in the younger-age group ( $0-10$ years) were more than those in the older-age group (10 years).

When the pedicle screw reaches $80 \%$ of the POCL, the fixation strength of the screw is sufficient. The mean POCL of $\mathrm{C} 3$ to $\mathrm{C} 7$ in children ranges from $25.54 \mathrm{~mm}$ to $28.13 \mathrm{~mm}$; therefore, it could be estimated that the lower cervical vertebrae could use $20.4 \mathrm{~mm}$ to $22.5 \mathrm{~mm}$ screw(s).

In this study, the $\mathrm{E}$ angle and the $\mathrm{F}$ angle were the angles between the pedicle axis and the vertebral sagittal/horizontal plane, respectively. However, these angles did not represent the best angle for the pedicle screw implantation, especially the $\mathrm{F}$ angle, which varied greatly within the same pedicle. Since the existence of the superior and inferior cartilage endplate in children's cervical vertebra and the vertebrae in the younger-age group still has the ossification centre, the selection of the screw implantation angle should avoid these important structures as much as possible.

\section{CONCLUSIONS}

In summary, the pedicle screw implantation for children under 4 years of age was feasible morphologically. However, the lower the age, the higher the risk; therefore, this surgery should be recommended for children over 7 years of age. Furthermore, because their vertebrae are still in the developing stage, the pedicle structures are still fine and fragile. In addition to this, the morphologies of different segments are different together with complex extension directions and important adjacent structures; thus, requiring higher surgical accuracy. It is essential to perform preoperative 3D observation and precisely design the screw channel to the pedicle of the child.

\section{Acknowledgments}

This work was funded by National Natural Science Foundation of China (30660072, 81260269, $81660358,81560348)$, Natural Science Foundation of Inner Mongolia, PRC (2009MS1112 \& 2012MS1149), Key projects of Inner Mongolia Medical University (No: YKD2015KJBW003). 


\section{REFERENCES}

1. Fujimori T, Yaszay B, Bartley CE, et al. Safety of pedicle screws and spinal instrumentation for pediatric patients: comparative analysis between 0 - and 5-year-old, 5- and 10-year-old, and 10- and 15-year-old patients. Spine. 2014; 39(7): 541-549, doi: 10.1097/BRS.0000000000000202, indexed in Pubmed: 24430718.

2. Geck MJ, Truumees E, Hawthorne D, et al. Feasibility of rigid upper cervical instrumentation in children: tomographic analysis of children aged 2-6. J Spinal Disord Tech. 2014; 27(3): E110-E117, doi: 10.1097/BSD.0b013e318291ce46, indexed in Pubmed: 23563351.

3. Hedequist $D$, Proctor M, Hresko $T$, et al. Screw fixation to $C 2$ in children: a case series and technical report. J Pediatr Orthop. 2009; 29(1): 21-25, doi:10.1097/ BPO.0b013e3181924367, indexed in Pubmed: 19098639.

4. Hedequist DJ. Modern posterior screw techniques in the pediatric cervical spine. World J Orthop. 2014; 5(2): 94-99, doi: 10.5312/wjo.v5.i2.94, indexed in Pubmed: 24829871.

5. Hong JT, Sung JH, Son BC, et al. Significance of laminar screw fixation in the subaxial cervical spine. Spine (Phila Pa 1976). 2008; 33(16): 1739-1743, doi: 10.1097/ BRS.0b013e31817d2aa2, indexed in Pubmed: 18628706.

6. Hwang SW, Gressot LV, Rangel-Castilla L, et al. Outcomes of instrumented fusion in the pediatric cervical spine. J Neurosurg Spine. 2012; 17(5): 397-409, doi: 10.3171/2012.8.SPINE12770, indexed in Pubmed: 22998404.

7. Lee H, Hong JT, Kim IIS, et al. Anatomic feasibility of posterior cervical pedicle screw placement in children: computerized tomographic analysis of children under 10 years old. J Korean Neurosurg Soc. 2014; 56(6): 475-481, doi: 10.3340/jkns.2014.56.6.475, indexed in Pubmed: 25628806.

8. Melcher RP, Puttlitz CM, Kleinstueck FS, et al. Biomechanical testing of posterior atlantoaxial fixation techniques. Spine. 2002; 27(22): 2435-2440, doi:10.1097/01.BRS.0000031262.05676.E0, indexed in Pubmed: 12435971.

9. Mendelsohn D, Dea N, Lee R, et al. Bilateral pedicle and crossed translaminar screws in C2. Asian Spine J. 2015; 9(5): 783-788, doi:10.4184/asj.2015.9.5.783, indexed in Pubmed: 26435799.

10. Menger RP, Storey CM, Nixon MKC, et al. Placement of C1 pedicle screws using minimal exposure: radiographic, clinical, and literature validation. Int J Spine Surg. 2015; 9: 43, doi: 10.14444/2043, indexed in Pubmed: 26484006.
11. Mueller TL, Miller NH, Baulesh DM, et al. The safety of spinal pedicle screws in children ages 1 to 12 . Spine J. 2013; 13(8): 894-901, doi:10.1016/j.spinee.2012.10.040, indexed in Pubmed: 23523435.

12. Platzer $P$, Jaindl $M$, Thalhammer $G$, et al. Cervical spine injuries in pediatric patients. J Trauma. 2007; 62(2): 389-96; discussion 394, doi:10.1097/01.ta.0000221802.83549.46, indexed in Pubmed: 17297330.

13. Polly DW, Potter BK, Kuklo T, et al. Volumetric spinal canal intrusion: a comparison between thoracic pedicle screws and thoracic hooks. Spine (Phila Pa 1976). 2004; 29(1): 63-69, doi: 10.1097/01.BRS.0000105525.06564.56, indexed in Pubmed: 14699278.

14. Rinella A, Cahill P, Ghanayem A, et al. Thoracic pedicle expansion after pedicle screw placement in a pediatric cadaveric vertebrae: a biomechanical analysis. Paper presented at: the SRS 39th Annual Meeting; Buenos Aires. 2004: Argentina.

15. Ruf M, Harms J. Posterior hemivertebra resection with transpedicular instrumentation: early correction in children aged 1 to 6 years. Spine. 2003; 28(18): 2132-2138, doi: 10.1097/01.BRS.0000084627.57308.4A, indexed in Pubmed: 14501925.

16. Ruf $M$, Harms J. Pedicle Screws in 1- and 2-Year-Old Children: Technique, Complications, and Effect on Further Growth. Spine. 2002; 27(21): E460-E466, doi: 10.1097/00007632-200211010-00019.

17. Sarwahi V, Sugarman EP, Wollowick AL, et al. Prevalence, Distribution, and Surgical Relevance of Abnormal Pedicles in Spines with Adolescent Idiopathic Scoliosis vs. No Deformity: A CT-Based Study. J Bone Joint Surg Am. 2014; 96(11): e92, doi: 10.2106/JBJS.M.01058, indexed in Pubmed:24897749.

18. Vara CS, Thompson GH. A cadaveric examination of pediatric cervical pedicle morphology. Spine. 2006; 31(10): 1107-1112, doi:10.1097/01.brs.0000216591.47505.17, indexed in Pubmed: 16648744.

19. Wang S, Qiu Y, Liu W, et al. The potential risk of spinal cord injury from pedicle screw at the apex of adolescent idiopathic thoracic scoliosis: magnetic resonance imaging evaluation. BMC Musculoskelet Disord. 2015; 16: 310, doi: 10.1186/s12891-015-0766-0, indexed in Pubmed: 26487645.

20. Zhang Z, Mu Z, Zheng W. Anterior pedicle screw and plate fixation for cervical facet dislocation: case series and technical note. Spine J. 2016; 16(1): 123-129, doi: 10.1016/j. spinee.2015.09.040, indexed in Pubmed: 26409419. 